\title{
Role of the Streptococcus gordonii SspB protein in the development of Porphyromonas gingivalis biofilms on streptococcal substrates
}

\author{
Richard J. Lamont, ${ }^{1}$ Azza El-Sabaeny, ${ }^{1}$ Yoonsuk Park, ${ }^{1}$ Guy S. Cook, ${ }^{2}$ \\ J. William Costerton ${ }^{3}$ and Donald R. Demuth ${ }^{4}$
}

\footnotetext{
1 Department of Oral Biology, Box 357132 University of Washington, Seattle, WA 98195, USA

2 Bacterin Inc., Bozeman, MT 59717, USA

3 Center for Biofilm Engineering, Montana State University, Bozeman, MT 59717, USA

4 Department of Biochemistry, University of Pennsylvania School of Dental Medicine, Philadelphia, PA 19104, USA
}

\author{
Author for correspondence: Richard J. Lamont. Tel: +1 206543 5477. Fax: +1 2066853162. \\ e-mail: lamon@u.washington.edu
}

\begin{abstract}
Porphyromonas gingivalis is an aggressive periodontal pathogen that persists in the mixed-species plaque biofilm on tooth surfaces. $P$. gingivalis cells attach to the plaque commensal Streptococcus gordonii and this coadhesion event leads to the development of $\boldsymbol{P}$. gingivalis biofilms. Binding of these organisms is multimodal, involving both the $\boldsymbol{P}$. gingivalis major fimbrial FimA protein and the species-specific interaction of the minor fimbrial Mfa1 protein with the streptococcal SspB protein. This study examined the contribution of the Mfa1-SspB interaction to $P$. gingivalis biofilm formation. $P$. gingivalis biofilms readily formed on substrata of $S$. gordonii DL1 but not on Streptococcus mutans cells which lack a coadhesion-mediating homologue of SspB. An insertional inactivation of the $\mathbf{m f a} 1 \mathrm{gene}$ in $P$. gingivalis resulted in a phenotype deficient in $S$. gordonii binding and unable to form biofilms. Furthermore, analysis using recombinant streptococci and enterococci showed that $P$. gingivalis biofilms formed on Enterococcus faecalis strains expressing SspB or translational fusions of SspB with SpaP (the non-adherent SspB homologue in S. mutans) containing the $P$. gingivalis adherence domain (SspB adherence region, BAR) of SspB. In contrast, an isogenic Ssp null mutant of S. gordonii DL1 was unable to support biofilm growth, even though this strain bound to $P$. gingivalis FimA at levels similar to wild-type $S$. gordonii DL1. Finally, site-specific mutation of two functional amino acid residues in BAR resulted in SspB polypeptides that did not promote the development of $P$. gingivalis biofilms. These results suggest that the induction of $P$. gingivalis biofilms on a streptococcal substrate requires functional SspB-minor fimbriae interactions.
\end{abstract}

Keywords : minor fimbriae, antigen I/II, coadhesion

\section{INTRODUCTION}

Dental plaque is a complex and dynamic biofilm that accumulates through the sequential and ordered colonization of over 500 different species of bacteria (Kolenbrander \& London, 1993; Marsh, 1994; Rosan \& Lamont, 2000). Plaque that comprises predominantly Gram-positive commensals such as Streptococcus gordonii and the related sanguis group of streptococci, and Actinomyces spp., can exist in the oral cavity in the

Abbreviations: $B A R, S s p B$ adherence region; CSLM, confocal scanning laser microscopy. absence of overt disease (Marsh, 1989, 1994; Rosan \& Lamont, 2000). However, population shifts within the plaque community that lead to over-representation of acidophiles or of Gram-negative anaerobes may contribute to the initiation and progression of the most common human oral diseases, caries and periodontal disease. Adult periodontitis is associated with subgingival plaque containing elevated levels of specific Gram-negative anaerobic bacteria, including Porphyromonas gingivalis (Socransky \& Haffajee, 1992). In addition to its niche in the subgingival biofilm, $P$. gingivalis has also been shown to invade gingival epithelial cells (Belton et al., 1999; Lamont et al., 1995) and to possess numerous virulence factors (e.g. pro- 
teases) that could contribute to periodontal tissue destruction and alveolar bone resorption (Curtis et al., 1999; Lamont \& Jenkinson, 1998, 2000; Travis et al., 2000).

Colonization of the plaque biofilm by $P$. gingivalis is contingent upon a variety of factors, including reduced oxygen tension, sufficient nutritional resources and an appropriate substrate for adhesion (Bradshaw et al., 1998; Lamont \& Jenkinson, 2000; Marsh, 1989, 1994). Furthermore, as a secondary colonizer of plaque, $P$. gingivalis is likely to encounter tissue surfaces that are already colonized with antecedent bacteria and their products. Consistent with these constraints, $P$. gingivalis has been shown to adhere to primary colonizing organisms such as S. gordonii and related streptococcal species, Actinomyces naeslundii, and to other Gramnegative secondary colonizing organisms such as Fusobacterium nucleatum, Treponema denticola and Bacteroides forsythus (Kinder \& Holt, 1989; Kolenbrander \& Andersen, 1989; Lamont et al., 1992; Schwarz et al., 1987; Yao et al., 1996). Coadhesion of $P$. gingivalis with these organisms involves specific adhesin-receptor interactions. For example, a galactose-specific lectin mediates adherence of $F$. nucleatum to $P$. gingivalis (Shaniztki et al., 1997). The coadhesion of $P$. gingivalis and $S$. gordonii is multimodal and involves at least two distinct adhesin-receptor pairs. The major and minor fimbriae of $P$. gingivalis both have been shown to bind to $S$. gordonii cells (Chung et al., 2000; Lamont et al., 1993). The major fimbriae, which may extend up to $3 \mu \mathrm{m}$ from the cell surface, are composed of the FimA protein (Yoshimura et al., 1984) and interact with a component of $S$. gordonii that has not yet been identified. In contrast, the minor fimbriae extend only $0 \cdot 1-0 \cdot 5 \mu \mathrm{m}$ from the cell surface (Hamada et al., 1996) and contribute to coadhesion by binding to the Ssp polypeptides (Brooks et al., 1997; Demuth et al., 2001). FimA and the minor fimbrial protein (designated Mfa1) are genetically and antigenically distinct (Arai et al., 2000), suggesting that these two adhesins differ both structurally and functionally.

The Ssp proteins are members of the antigen I/II family of streptococcal surface proteins that are highly conserved in overall structure and primary sequence across all the human oral streptococcal species (Jenkinson \& Demuth, 1997). However, despite the high degree of structural similarity, $P$. gingivalis adheres to streptococci and interacts with antigen I/II proteins in a speciesspecific manner. Our previous studies have shown that $P$. gingivalis adheres avidly to the $\mathrm{SspA}$ and $\mathrm{SspB}$ polypeptides of $S$. gordonii, but does not interact with the SpaP protein, the homologue of SspA/B expressed by Streptococcus mutans (Brooks et al., 1997). Adherence is mediated by a discrete domain, designated BAR ( $\operatorname{spp} \underline{B}$ adherence region), comprising amino acid residues 1167-1250 of SspB, which is fully conserved between SspB and SspA (Brooks et al., 1997; Demuth et al., 2001). Within BAR, Asn ${ }^{1182}$ and $\mathrm{Val}^{1185}$ have been suggested to confer a unique secondary structural motif that is recognized and bound by the $P$. gingivalis minor fimbrial protein (Demuth et al., 2001). This structural motif (and $\mathrm{Asn}^{1182}$ and $\mathrm{Val}^{1185}$ ) is essential for adherence and is not conserved in SpaP polypeptide.

Using an open flow chamber under conditions of low shear force, Cook et al. (1998) showed that subsequent to its adherence to $S$. gordonii, $P$. gingivalis rapidly accretes to form a biofilm consisting of structures resembling towering microcolonies separated by fluidfilled channels. Although adherence is clearly a first step that is necessary for accretion to occur, it is not known how the two independent fimbrial-mediated adherence mechanisms of $P$. gingivalis contribute to biofilm formation. In this study, we show that the formation of $P$. gingivalis biofilms exhibits the same species specificity observed in minor-fimbriae-mediated adherence, suggesting that the Mfa1-mediated interaction of $P$. gingivalis with the streptococcal Ssp polypeptides may drive biofilm development. Moreover, $P$. gingivalis biofilms do not form on an Ssp null mutant of S. gordonii which retains the ability to interact with FimA, or when the $m f a 1$ gene of $P$. gingivalis has been disrupted. In addition, recombinant Enterococcus faecalis strains expressing chimeric $\mathrm{SspB} / \mathrm{SpaP}$ proteins containing BAR support biofilm growth, whereas hybrid proteins without BAR do not. SspB proteins containing sitespecific mutations of essential functional amino acid residues of BAR do not permit $P$. gingivalis biofilm development. These results suggest that the interaction of the minor fimbriae with Ssp is sufficient to allow $P$. gingivalis to form biofilms on a streptococcal substrate.

\section{METHODS}

Bacteria and culture conditions. Bacterial strains used in this study are listed in Table 1. $P$. gingivalis strains were cultured in Trypticase soy broth (BBL) supplemented with $1 \mathrm{mg}$ yeast extract $\mathrm{ml}^{-1}, 5 \mu \mathrm{g}$ haemin $\mathrm{ml}^{-1}$ and $1 \mu \mathrm{g}$ menadione $\mathrm{ml}^{-1}$, under anaerobic conditions $\left(85 \% \mathrm{~N}_{2}, 10 \% \mathrm{H}_{2}, 5 \% \mathrm{CO}_{2}\right)$ at $37^{\circ} \mathrm{C}$ overnight. Erythromycin at $10 \mu \mathrm{g} \mathrm{ml}^{-1}$ was added to the medium when necessary. For radio-labelling, $\left[{ }^{3} \mathrm{H}\right]$ thymidine was added to the culture medium at $3.7 \times 10^{5} \mathrm{~Bq} \mathrm{~m}^{-1}$. Streptococci and enterococci were grown in Trypticase peptone broth (BBL) supplemented with $5 \mathrm{mg}$ yeast extract $\mathrm{ml}^{-1}$ and $0.5 \%$ glucose as carbon source, at $37^{\circ} \mathrm{C}$ under static conditions. S. gordonii OB219 is a derivative of strain DL1 in which both the $s s p A$ and $s s p B$ genes are insertionally inactivated (Demuth et al., 1996; Jenkinson et al., 1993) and was a kind gift from Dr Howard Jenkinson, University of Bristol.

Construction of recombinant $E$. faecalis strains. E. faecalis SspB expresses full-length SspB peptide encoded by the $S$. gordonii sspB gene (Demuth et al., 1989). E. faecalis strains Spac4 and Spac5 express the hybrid SspB/SpaP peptides Spac4 and Spac5 derived from a fusion of specific portions of the $S$. gordonii sspB and the $S$. mutans spaP genes. Construction of these strains has been reported previously (Demuth et al., 2001). As shown schematically in Fig. 1, Spac5 differs from Spac4 only in that it contains the portion of SspB representing BAR. E. faecalis NG expresses full-length SspB protein that possesses a glycine substitution for asparagine at position 1182 in BAR. Similarly, E. faecalis VP expresses full-length SspB 
Table 1. Bacterial strains

\begin{tabular}{|c|c|c|}
\hline Strain & Relevant characteristics* & Source or reference \\
\hline P. gingivalis 33277 & Type strain from ATCC & Laboratory stock \\
\hline$P$. gingivalis SMF1 & Derivative of 33277 with an insertional inactivation of the $m f a 1$ gene; $\mathrm{Em}^{\mathrm{R}}$ & This study \\
\hline S. gordonii DL1 & Wild-type & Laboratory stock \\
\hline S. gordonii OB219 & Derivative of DL1, sspAB::ermAM; Em ${ }^{\mathrm{R}}$ & Demuth et al. (1996) \\
\hline S. mutans KPSK2 & Clinical isolate & Laboratory stock \\
\hline E. faecalis S161 & Wild-type & Laboratory stock \\
\hline E. faecalis 401 & Derivative of S161 containing pAM401; $\mathrm{Cm}^{\mathrm{R}}$ & Demuth et al. (1989) \\
\hline E. faecalis $\mathrm{SspB}$ & Derivative of $\mathrm{S} 161$ expressing $S$. gordonii $\mathrm{SspB} ; \mathrm{Cm}^{\mathrm{R}}$ & Demuth et al. (1989) \\
\hline E. faecalis Spac4 & Derivative of S161 expressing hybrid SspB/SpaP polypeptide Spac4; $\mathrm{Cm}^{\mathrm{R}}$ & Demuth et al. (2001) \\
\hline E. faecalis Spac5 & Derivative of S161 expressing hybrid SspB/SpaP polypeptide Spac5; Cm ${ }^{\mathrm{R}}$ & Demuth et al. (2001) \\
\hline E. faecalis NG & Derivative of S161 expressing SspB containing $N / G^{1182}$ substitution; $\mathrm{Cm}^{\mathrm{R}}$ & Demuth et al. (2001) \\
\hline E. faecalis VP & Derivative of S161 expressing SspB containing V/ $\mathrm{P}^{1185}$ substitution; $\mathrm{Cm}^{\mathrm{R}}$ & Demuth et al. (2001) \\
\hline E. faecalis DM & $\begin{array}{l}\text { Derivative of } \mathrm{S} 161 \text { expressing } \mathrm{SspB} \text { containing both } \mathrm{N} / \mathrm{G}^{1182} \text { and } \mathrm{V} / \mathrm{P}^{1185} \\
\text { substitutions; } \mathrm{Cm}^{\mathrm{R}}\end{array}$ & Demuth et al. (2001) \\
\hline
\end{tabular}

*ATCC, American Type Culture Collection, Manassas, VA, USA; $\mathrm{Em}^{\mathrm{R}}, \mathrm{Cm}^{\mathrm{R}}$, resistance to erythromycin and chloramphenicol respectively.
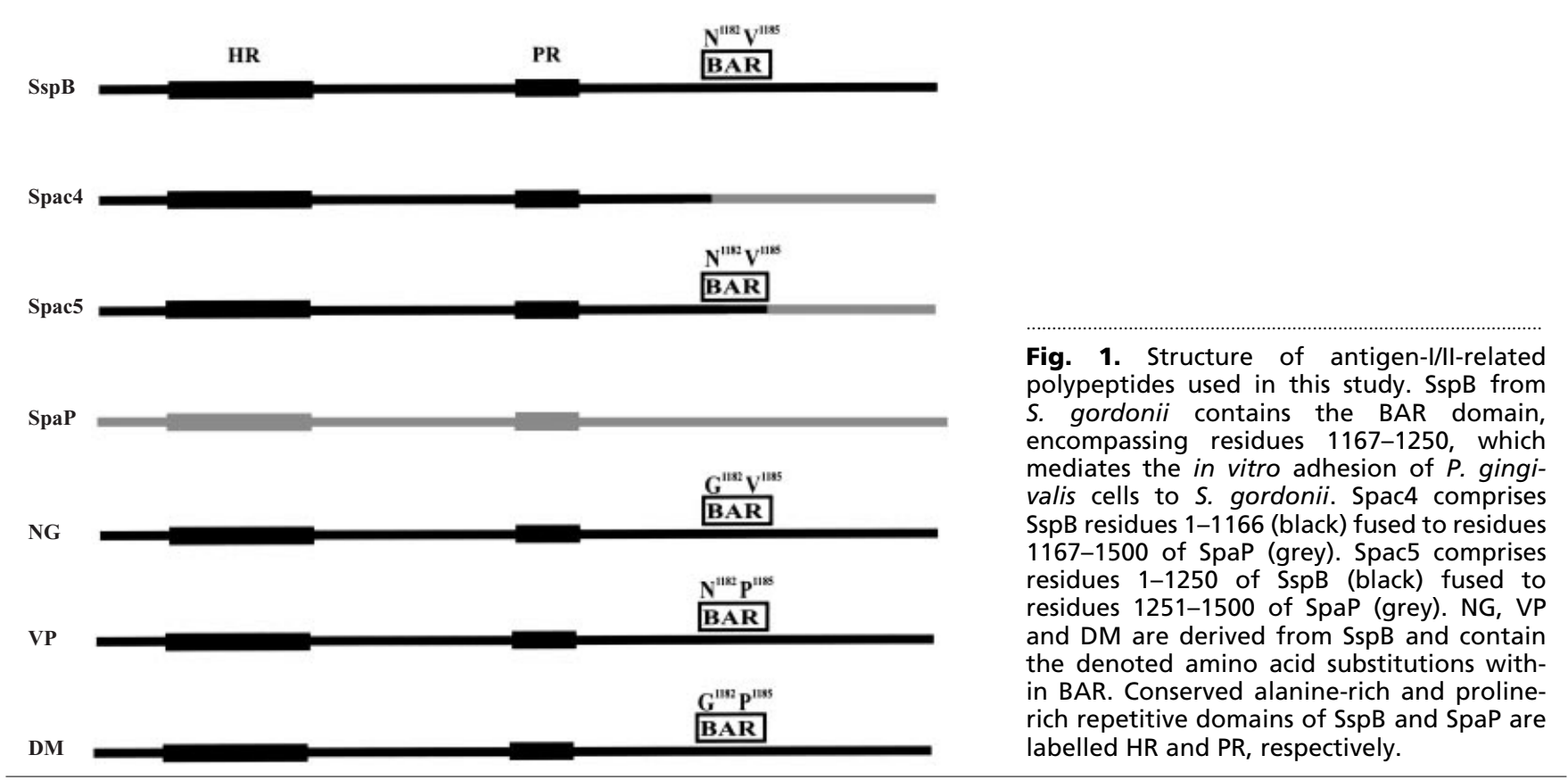

Fig. 1. Structure of antigen-I/II-related polypeptides used in this study. SspB from S. gordonii contains the BAR domain, encompassing residues 1167-1250, which mediates the in vitro adhesion of $P$. gingivalis cells to $S$ gordonii Spac4 comprises (black) fused to residues (grey). Spac5 comprises in BAR. Conserved alanine-rich and prolinerich repetitive domains of SspB and SpaP are .

containing a proline residue replacing the native valine at position 1185 in BAR, and E. faecalis DM expresses SspB containing both Asn/ $/ \mathrm{Gly}^{1182}$ and $\mathrm{Val} / \mathrm{Pro}^{1185}$ substitutions (Fig. 1). Site-specific mutations were introduced into $s s p B$ using the QuikChange Site Directed Mutagenesis Kit (Stratagene) and the appropriate forward and reverse oligonucleotide primers. Conditions for the generation of the specific mutations in the $s s p B$ gene and the sequences of the oligonucleotide primers were described previously (Demuth et al., 2001). All gene constructs were transferred into the E. faecalisEscherichia coli shuttle vector, pAM401 (Wirth et al., 1986) and transformed into E. faecalis S161 by electroporation. E. faecalis S161 does not express an endogenous homologue of antigen I/II. Recombinant colonies were selected on the appropriate agar plates containing $15 \mu \mathrm{g}$ chloramphenicol $\mathrm{ml}^{-1}$. Chimeric gene constructs were confirmed by restriction mapping. The site-specific mutations were confirmed by nucleotide sequencing at the University of Pennsylvania Automated Sequencing Facility.

ELISA of fimbrillin binding. Purified recombinant fimbrillin (FimA) was produced from the $P$. gingivalis fimA gene in the pET30 expression system as described previously (Xie et al., 2000). For ELISA experiments, S. gordonii DL1 or OB219 cells $\left(10^{7}\right)$ were deposited onto microtitre plate wells. After blocking with $3 \%$ BSA and washing, bacterial cells were reacted $\left(1 \mathrm{~h}, 37^{\circ} \mathrm{C}\right)$ sequentially with varying concentrations of FimA, specific FimA antibodies (Xie et al., 2000), diluted $1: 10000$, and alkaline phosphatase-conjugated anti-rabbit IgG. Binding reactions were detected with $p$-nitrophenyl phosphate and $A_{405}$ was recorded. Controls without strepto- 
coccal cells or without FimA were included in all experiments and were less than $10 \%$ of specific values.

Construction of $\boldsymbol{P}$. gingivalis minor-fimbriae-deficient mutant. $P$. gingivalis SMF1 was created by homologous recombination between $P$. gingivalis 33277 chromosomal DNA and a suicide plasmid carrying an internal fragment of the $m f a 1$ gene as follows. A $0.85 \mathrm{~kb}$ internal fragment of $m f a 1$ was amplified by PCR with the primers 5'-TATCCGAGGCCAATGCTATC-3' and $5^{\prime}$-GCATCAAGAAGTTGGGCTTC-3'. Amplification conditions were denaturation at $94{ }^{\circ} \mathrm{C}$ for $30 \mathrm{~s}$, annealing at $55^{\circ} \mathrm{C}$ for $30 \mathrm{~s}$ and elongation at $72{ }^{\circ} \mathrm{C}$ for $3 \mathrm{~min}$, for 30 cycles. The product was cloned into the PCR cloning vector pCR11 (Invitrogen). A Bam HI-XbaI fragment was excised and ligated into the shuttle vector pVA3000 that contains the antibiotic resistance cassette ermAM-ermF (Lee et al., 1996). The resulting plasmid was conjugated into $P$. gingivalis 33277 as described previously (Park \& Lamont, 1998). Transconjugants with erythromycin resistance were selected. Confirmation that the plasmid had integrated into the $m f a 1$ gene was achieved by Southern blotting and loss of mRNA expression was established by RT-PCR using the primers defined above.

Interbacterial binding assay. Coadhesion between P. gingivalis and S. gordonii was measured with the nitrocellulose blot assay described previously (Lamont et al., 1992). Briefly, $S$. gordonii cells were suspended in buffered $\mathrm{KCl}(5 \mathrm{mM} \mathrm{KCl}$, $\left.2 \mathrm{mM} \mathrm{K}_{2} \mathrm{PO}_{4}, 1 \mathrm{mM} \mathrm{CaCl} 2, \mathrm{pH} 6.0\right)$ and $10^{8}$ bacteria were deposited on nitrocellulose paper in a dot-blot apparatus. The blot was washed three times in $\mathrm{KCl}$ containing $0 \cdot 1 \%$ Tween 20 (KCl-Tween). The adsorbed bacteria were subsequently incubated for $2 \mathrm{~h}$ at room temperature with $\left[{ }^{3} \mathrm{H}\right]$ thymidinelabelled $P$. gingivalis strains 33277 or SMF1 (mean c.p.m. per cell, $5 \times 10^{-4}$ ) suspended in $\mathrm{KCl}$-Tween. After washing to remove unbound organisms, the experimental areas of the nitrocellulose were excised and adherence was quantified by scintillation spectroscopy.

Biofilm formation. Biofilm studies were carried out essentially as described by Cook et al. (1998). Briefly, standard glass coverslips were coated with centrifugation-clarified pooled human saliva for $30 \mathrm{~min}$ at $37^{\circ} \mathrm{C}$ and washed three times with sterile PBS. Saliva-coated glass coverslips were mounted under aseptic conditions in a polycarbonate flow cell $(0.6 \times 1.0 \mathrm{~cm})$ with an attached peristaltic pump. Flow cells were inoculated initially with the streptococcal or enterococcal strains $\left(10^{7}\right.$ cells $\mathrm{ml}^{-1}$ ) at a flow rate of $4 \cdot 1 \mathrm{ml} \mathrm{h}^{-1}$ for $2 \mathrm{~h}$ (S. gordonii) or $4 \mathrm{~h}$ (S. mutans and E. faecalis). S. mutans and E. faecalis adhere less efficiently to saliva surfaces in comparison to $S$. gordonii, hence the longer incubation period. The formation of a $70-80 \%$ confluence bacterial monolayer was confirmed by phase-contrast microscopy. The flow cells were then subjected to a secondary inoculation of $P$. gingivalis $\left(10^{7}\right.$ cells $\mathrm{ml}^{-1}$ ) under similar flow conditions. The total length of exposure of streptococci or enterococci to $P$. gingivalis was $4 \mathrm{~h}$, which is less than the doubling time for $P$. gingivalis cells when grown in rich broth. Thus, under these conditions the accumulation of $P$. gingivalis biofilms is a result of accretion of planktonic cells rather than growth of bound cells. Biofilms were visualized with a Bio-Rad MRC600 confocal scanning laser microscope with an Olympus IMT-2 inverted light microscope and a MS plan $60 \times 1.4 \mathrm{NA}$ objective. For confocal scanning laser microscopy (CSLM), streptococci and enterococci were stained with $10 \mathrm{mg}$ hexidium iodide $\mathrm{ml}^{-1}$ and $P$. gingivalis was stained with $10 \mathrm{mg}$ fluorescein $\mathrm{ml}^{-1}$. CSLM was first used in the reflected white light mode to directly observe biofilm formation over the course of the experiments. A representative area of the coverslip was then selected and observed under reflected laser light of 488, 546 and $647 \mathrm{~nm}$. A series of fluorescent optical sections were collected to determine the depth of the bacterial layers and/or microcolonies and to assemble a three-dimensional view of the biofilms using the Slicer (Fortner Research) imaging program. Under these conditions of biofilm formation, $P$. gingivalis cells were observed to gradually and continuously accumulate on the substratum over the $4 \mathrm{~h}$ inoculation period. Sloughing and reattachment of biofilm microcolonies was not observed and no significant auto-aggregation of planktonic $P$. gingivalis cells occurred.

\section{RESULTS}

\section{Species specificity of $\boldsymbol{P}$. gingivalis biofilm formation}

The adherence of $P$. gingivalis minor fimbriae with streptococci is specific for the antigen I/II proteins $(\mathrm{Ssp})$ expressed by $S$. gordonii and does not occur with related antigen I/II polypeptides (SpaP) of $S$. mutans cells (Brooks et al., 1997). To determine if biofilm formation exhibits a similar species specificity, we tested the ability of $P$. gingivalis to accumulate on substrata of $S$. gordonii or $S$. mutans attached to saliva-coated glass coverslips in a flow cell. Planktonic $P$. gingivalis cells adhered rapidly to the $S$. gordonii DL1 substratum and accumulated progressively over the $4 \mathrm{~h}$ inoculation period to form large towering microcolonies (Fig. 2a). In contrast, no accumulation occurred on a substratum composed of $S$. mutans KPSK2 cells (Fig. 2b) or with an isogenic mutant of $S$. gordonii DL1 (strain OB219) that does not express antigen I/II (Fig. 2c). The P. gingivalis microcolonies that formed on $S$. gordonii DL1 routinely accumulated to depths of $60-80 \mu \mathrm{m}$, whereas the streptococcal substratum in the absence of $P$. gingivalis cells was approximately $5-10 \mu \mathrm{m}$ in depth. P. gingivalis did not accumulate into microcolonies on a saliva-coated coverglass in the absence of streptococci (Fig. 2d) and no large aggregates of planktonic $P$. gingivalis cells were observed in the flow-cell inoculum. Thus, the development of $P$. gingivalis biofilms exhibits a species specificity similar to that previously observed for minor-fimbriae-mediated adhesion, suggesting that the interaction of the minor fimbriae with the appropriate antigen I/II polypeptide drives the formation of $P$. gingivalis biofilms on streptococci.

\section{Minor-fimbriae-mediated adherence is required for $P$. gingivalis biofilm development}

To further investigate the role of the interaction of Mfa1, the minor fimbrial protein, with $S \mathrm{spB}$ of $S$. gordonii in biofilm formation, we examined the development of biofilms on E. faecalis $\mathrm{SspB}$, a recombinant enterococcal strain expressing $\mathrm{SspB}$. As shown in Fig. 3(a), $P$. gingivalis adhered and accumulated on the E. faecalis $\mathrm{SspB}$ substratum and formed microcolonies that were similar to the structures that formed on substrates of $S$. gordonii DL1. In contrast, no accretion of $P$. gingivalis occurred on a substratum of the parent E. faecalis strain 401 that contains the pAM401 vector without an insert and does not express SspB (Fig. 3b). 

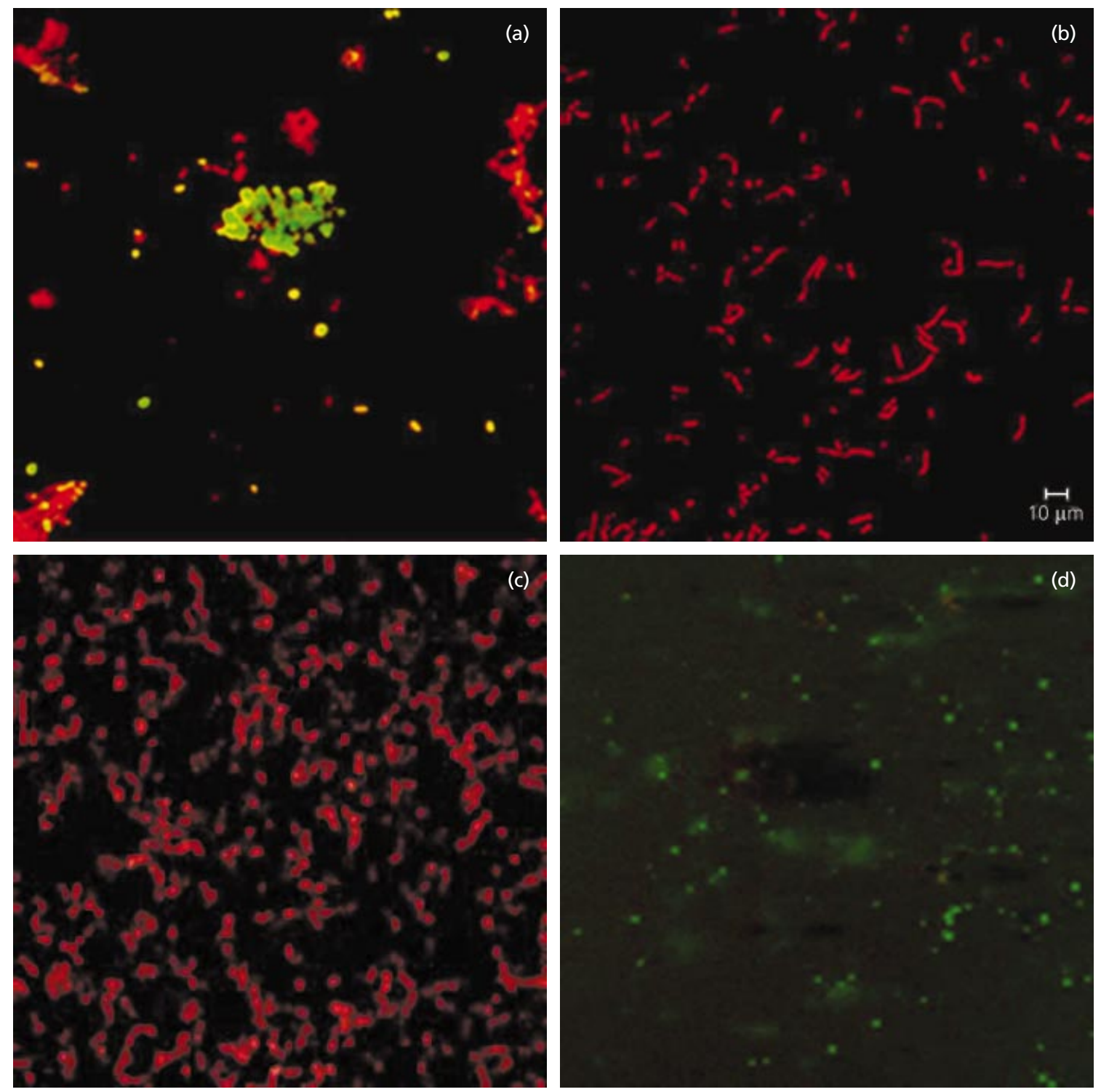

Fig. 2. CSLM of biofilm development on saliva-coated glass in flow cells. (a) $P$. gingivalis cells (green) adhere and develop a biofilm microcolony on S. gordonii (red). Co-localized bacteria appear yellow. (b) $P$. gingivalis does not form a biofilm on S. mutans cells (red), or (c) on the Ssp-deficient mutant S. gordonii OB219 (red). (d) In the absence of S. gordonii, $P$. gingivalis cells (green) do not form biofilm microcolonies on the saliva-conditioned substratum.

To exclude the possibility that inactivation of antigen I/II expression in OB219 may also influence majorfimbriae-mediated adherence of $P$. gingivalis to $S$. gordonii, we examined the binding of purified recombinant FimA protein to OB219 cells. As shown in Fig. 4, recombinant FimA protein bound to $S$. gordonii OB219 in a dose-dependent manner and overall binding was similar to that observed with the parent organism, $S$. gordonii DL1, which expresses antigen I/II. Thus, while $S$. gordonii OB219 is capable of interacting with the major fimbriae of $P$. gingivalis, this interaction is not sufficient to induce biofilm formation. Another potentially confounding factor in interpretation of these results would be binding of $\mathrm{SspB}$ to additional molecules on the surface of $P$. gingivalis. To address this possi- bility, an Mfa1-deficient mutant of $P$. gingivalis was constructed. In an interbacterial binding assay, mutant strain SMF1 demonstrated a reduction in binding to S. gordonii of up to $80 \%$ in comparison to the parent strain (Fig. 5). Furthermore, SMF1 was defective in biofilm formation with $S$. gordonii and with $E$. faecalis SspB (not shown). Collectively, these results support the concept that minor-fimbriae-mediated adherence is required for $P$. gingivalis biofilm formation.

\section{BAR is sufficient to promote development of P. gingivalis biofilms}

To confirm the role of minor fimbriae SspB-mediated adherence in biofilm development, we determined if the 

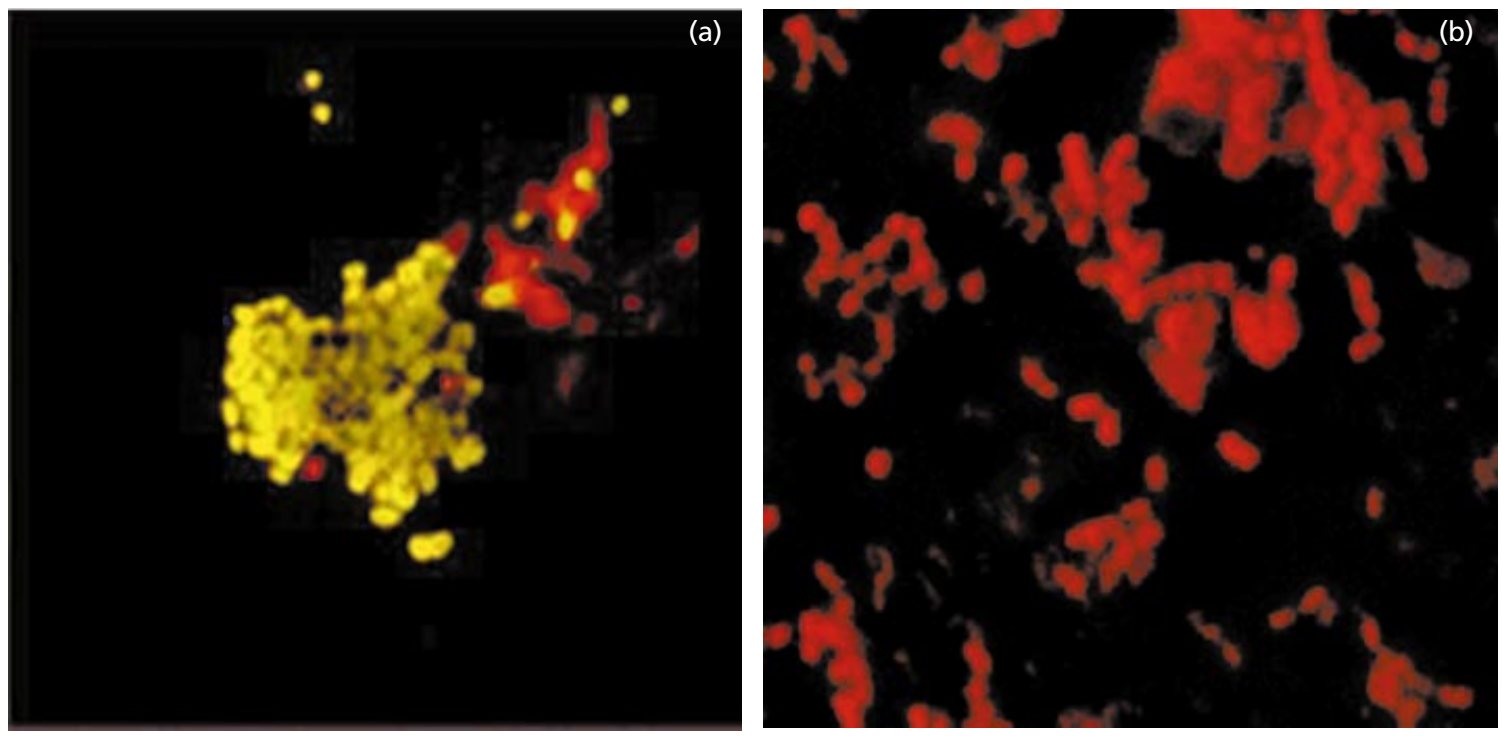

Fig. 3. CSLM of biofilm development on saliva-coated glass in flow cells. (a) $P$. gingivalis cells (green/yellow) adhere and develop a biofilm microcolony on $E$. faecalis SspB (red) which expresses functional SspB protein. (b) In contrast, $P$. gingivalis does not form biofilms on E. faecalis 401 (red) which does not express SspB.

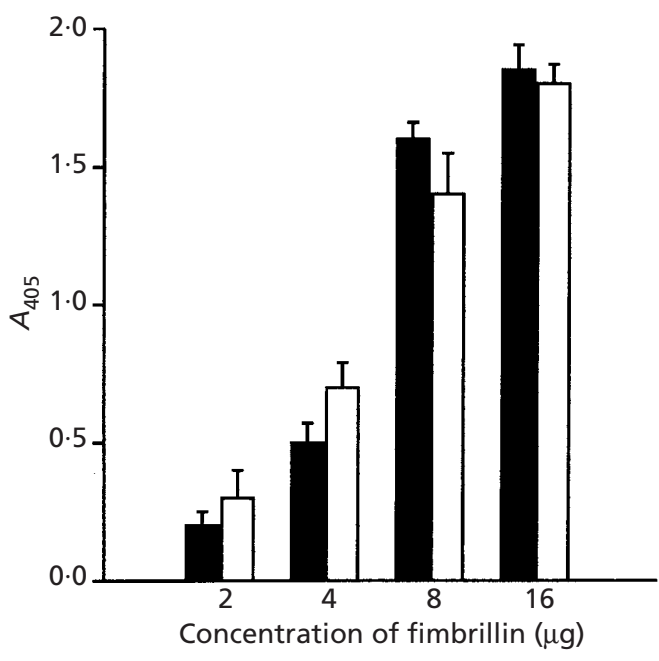

Fig. 4. ELISA of recombinant fimbrillin binding to $S$. gordonii strains DL1 (black bars) or OB219 (white bars). Error bars represent standard deviation $(n=3)$.

BAR region of SspB was sufficient to promote the development of $P$. gingivalis biofilms. To accomplish this, we monitored the formation of $P$. gingivalis biofilms on E. faecalis strains expressing chimeric $\mathrm{SspB} / \mathrm{SpaP}$ polypeptides and on strains expressing SspB that contained site-specific mutations of functional amino acid residues of BAR. Chimeric proteins Spac4 and Spac5 (see Methods) contain residues 1-1167 and 1-1250 of SspB, respectively, fused to the appropriate C-terminal sequences of SpaP. Thus, Spac5 differs from Spac4 only in that it contains BAR. As shown in

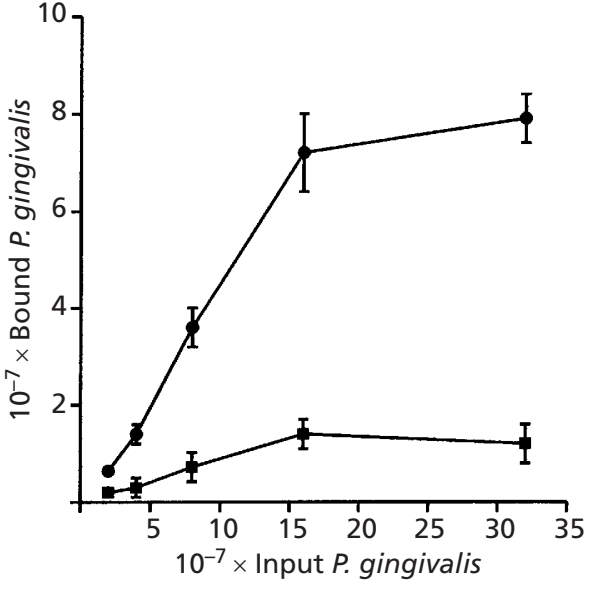

Fig. 5. Adhesion of $P$. gingivalis 33277 (O) and its minorfimbriae-deficient mutant, SMF1 ( $\square$ ), to cells of S. gordonii DL1. Error bars represent standard deviation $(n=3)$.

Fig. 6(a), P. gingivalis accumulated on immobilized E. faecalis Spac5 cells, forming microcolonies similar in structure and depth to those observed on E. faecalis SspB and on $S$. gordonii DL1. In contrast, $P$. gingivalis cells did not accumulate on E. faecalis Spac4 (Fig. 6b) or on E. faecalis strains expressing $S \mathrm{spB}$ polypeptides in which $\mathrm{Asn}^{1182}$ and $\mathrm{Val}^{1185}$ were substituted with the corresponding residues of SpaP, Gly ${ }^{1182}$ and Pro $^{1185}$ (not shown). These results show that $P$. gingivalis biofilms do not form in the absence of the SspB BAR domain. In addition, when specific amino acid residues of the BAR 

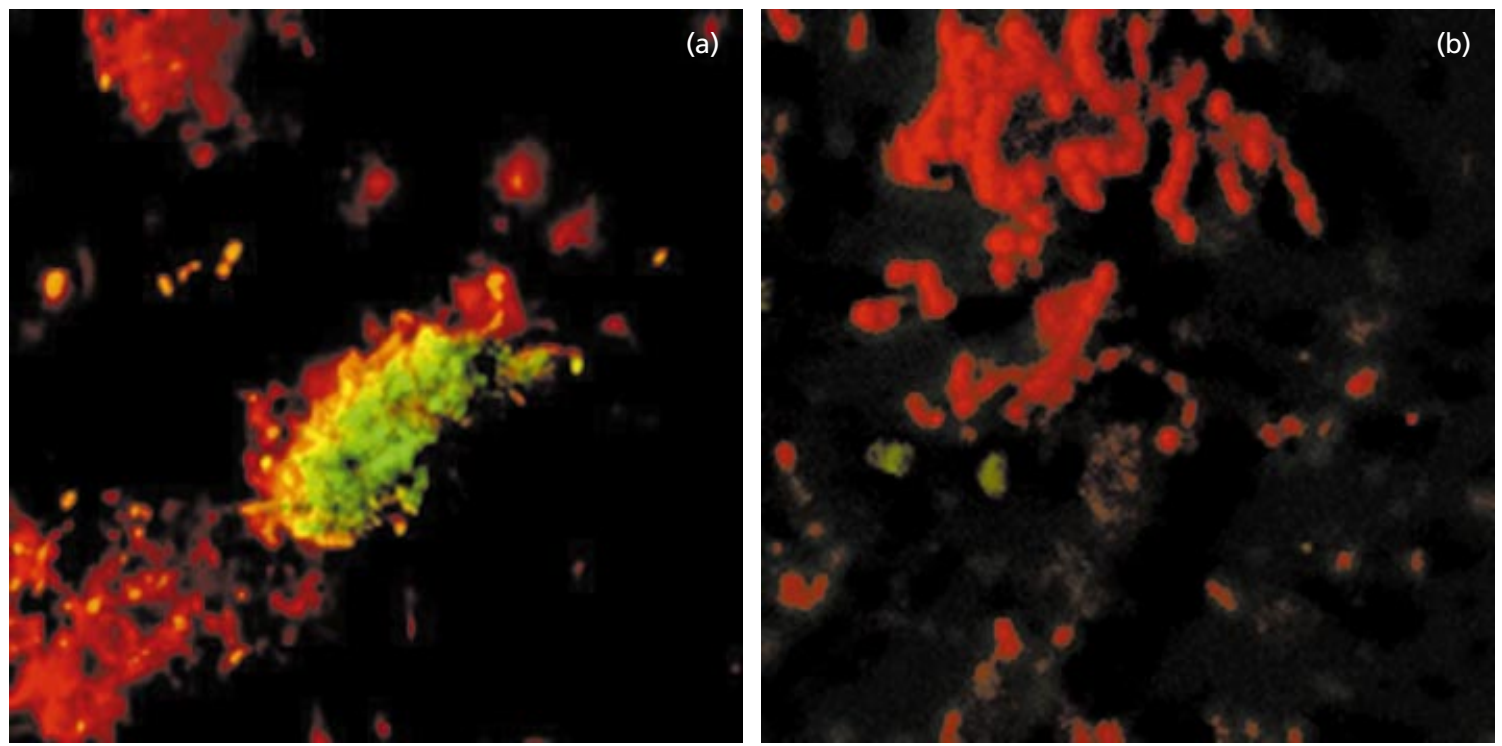

Fig. 6. CSLM of biofilm development on saliva-coated glass in flow cells. (a) $P$. gingivalis cells (green/yellow) adhere and develop a biofilm microcolony on E. faecalis Spac5 (red) which expresses a chimeric SspB/SpaP polypeptide possessing the BAR domain. (b) P. gingivalis cells do not develop into biofilms on E. faecalis Spac4 (red) which expresses a chimeric SspB/SpaP polypeptide that does not possess the BAR domain.

region of $S \mathrm{spB}$ are altered to the corresponding residues of the S. mutans SpaP polypeptide, then biofilm formation is impeded.

\section{DISCUSSION}

The human oral cavity represents a hostile environment for micro-organisms due to the presence of multiple antimicrobial activities and the constant flow of saliva across tissue surfaces (Marsh, 1989; Rosan \& Lamont, 2000; Scannapieco, 1994). Oral bacteria insulate themselves from these adverse conditions by adhering tightly to oral tissues and to other oral organisms to form a complex multispecies biofilm. The initial colonization of the plaque biofilm by $P$. gingivalis may thus be facilitated by its adhesion to other bacteria such as F. nucleatum (Bradshaw et al., 1998; Kinder \& Holt, 1989; Kolenbrander \& Andersen, 1989) and specific oral streptococci such as S. gordonii (Lamont et al., 1992). However, the initial attachment to antecedent bacteria in plaque represents only the first step in the process by which $P$. gingivalis becomes established and persists in the oral ecosystem. The subsequent expansion of $P$. gingivalis populations in plaque is likely to be dependent upon nutritional and environmental factors (Guggenheim et al., 2001; Marsh, 1994) along with the ability of adherent $P$. gingivalis to recruit additional planktonic cells into the developing sessile biofilm (Cook et al., 1998).

Our studies have focused on the adherence of $P$. gingivalis to streptococci and the subsequent formation of biofilms on the streptococcal substrate. Adherence of $P$. gingivalis is mediated by at least two distinct adhesin-receptor pairs (Brooks et al., 1997; Chung et al., 2000; Lamont et al., 1993) involving both the FimA and Mfa1 proteins, the predominant polypeptides composing the major and minor fimbriae of $P$. gingivalis, respectively. Both adhesins have been shown to mediate adherence to $S$. gordonii under static conditions in vitro. Several lines of evidence from the present investigation suggest that the development of $P$. gingivalis biofilms on a $S$. gordonii substratum is dependent primarily on the interaction of minor fimbriae with streptococcal antigen I/II. First, the development of $P$. gingivalis biofilms exhibited the same species specificity as was previously demonstrated for minor-fimbriae-mediated adherence to streptococci in vitro. Second, biofilms did not develop on S. gordonii OB219, a strain that does not express the antigen I/II receptor for the minor fimbrial adhesin but which retains the ability to interact with the major fimbrial adhesin. Thus, FimA-mediated adhesion alone is insufficient to induce the formation of biofilms. Third, a minor-fimbriae-deficient mutant of $P$. gingivalis was impaired in its ability to bind to $S$. gordonii and was unable to form biofilms. The structural motif (BAR) of antigen I/II that is recognized by Mfa1 also was shown to be essential for the development of $P$. gingivalis biofilms. Recombinant E. faecalis strains expressing chimeric $\mathrm{SspB} / \mathrm{SpaP}$ proteins lacking BAR or expressing SspB site-specific mutants lacking essential residues of BAR did not support biofilm development. These results demonstrate that wild-type $P$. gingivalis $\left(\mathrm{FimA}^{+}, \mathrm{Mfa}^{+}\right.$) are unable to form biofilms on streptococci or enterococcal constructs that do not possess the appropriate antigen I/II determinants, even if the cells are capable of interacting with FimA. Thus, individual components of the multimodal binding interaction appear to have 


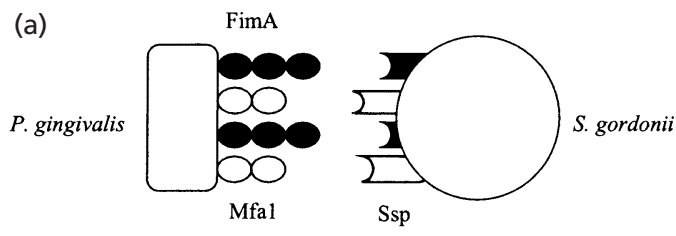

(b)

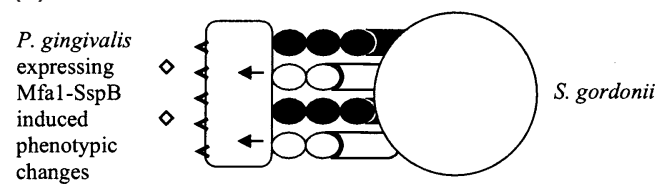

(c)

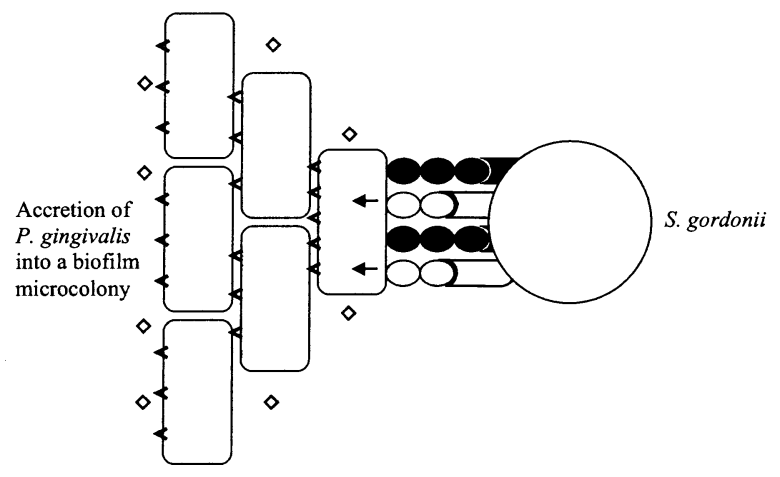

Fig. 7. Schematic model of events contributing to the formation of a $P$. gingivalis biofilm on immobilized $S$. gordonii cells. (a) The initial association of $P$. gingivalis with $S$. gordonii is mediated by the interaction of the major fimbriae, comprised of the FimA protein ( $)$ with an unidentified receptor on the $S$. gordonii cell surface ( $\boldsymbol{Z}$ ). (b) Higher-affinity binding of $P$. gingivalis to $S$. gordonii occurs as FimA and Mfa1 $(O)$ engage

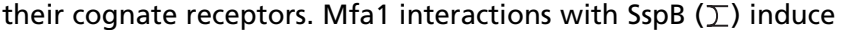
signalling events $(\rightarrow)$ within $P$. gingivalis that lead to phenotypic changes, potentially alteration of surface receptors $(<)$ and/or expression of extracellular signalling molecules $(\diamond)$. (c) Planktonic cells adhere to the immobilized $P$. gingivalis cells and accrete into a biofilm microcolony.

discrete and independent roles. Consistent with this concept, Love et al. (2000) found FimA binding to $S$. gordonii to be independent of SspA or SspB and that a FimA mutant was able to co-invade dentinal tubules with $S$. gordonii.

While our results indicate that the Mfa1-SspB-mediated interaction is necessary for biofilm development, they do not completely exclude the possibility of a role for the other adhesin-receptor pairs that participate in the multimodal $P$. gingivalis $-S$. gordonii coadhesion event. These molecules could serve either to allow initial lowaffinity contact between the organisms or to help stabilize the interaction between adhered organisms. The long major fimbriae that can extend up to $3 \mu \mathrm{m}$ from the cell surface would be suitable candidates to effect relatively long-range, low-affinity interactions with the streptococci. The proximal association between the two organisms would then allow the close range, higher affinity Mfa1-SspB interactions that induce biofilm formation. The role of the major fimbriae will be more apparent when the identification and distribution of their cognate receptor is accomplished. This model of $P$. gingivalis-S. gordonii coadhesion and biofilm development is represented in Fig. 7. Longer distance interactions, primarily mediated by the major fimbriae, facilitate localization of cells of $P$. gingivalis and $S$. gordonii. Multimodal coadhesion then occurs involving both Mfa1-SspB and FimA-receptor interactions. The engagement of Mfa1 with $\mathrm{SspB}$ initiates a signal transduction event within $P$. gingivalis that results in cells that are primed for biofilm formation. Theoretically, the transformation to a biofilm ready state could entail expression of a surface receptor that permits autoaggregation of $P$. gingivalis and stimulates other $P$. gingivalis cells to produce the same receptor. Alternatively, or additionally, a soluble signalling molecule may be released by $P$. gingivalis that stimulates cells to accumulate on the solid phase.

Biofilm formation by other organisms such as Pseudomonas aeruginosa requires cell-to-cell signalling through quorum sensing (Davies et al., 1998). P. gingivalis does not possess homoserine lactone-based signalling systems that are used by Pseudomonas aeruginosa for biofilm differentiation; however, $P$. gingivalis does have a functional LuxS-based quorum sensing system (Chung et al., 2001). The role of LuxS in P. gingivalis biofilms remains to be determined as does the possibility that signalling molecules produced by the streptococci or by E. faecalis are also involved in the biofilm process.

The maintenance of the complex oral biofilm community is thought to depend on a series of cohesive interactions that stabilize plaque structure and facilitate nutritional interrelationships (Kolenbrander \& London, 1993 ; Rosan \& Lamont, 2000). Moreover, the adherence event itself can transduce information into the bacterial cell regarding the substrate and environment in which the organism has localized (Cleary \& Retnoningrum, 1994; Cornelis \& Van Gijsegem, 2000; McNab \& Jenkinson, 1998). In the case of BAR-mediated adhesion, $P$. gingivalis responds by accretion into microcolonies. This process can be postulated to involve intracellular signalling pathways within $P$. gingivalis, an example of which has recently been described (Hayashi et al., 2000), and modulation of gene expression. The complete range of phenotypic changes that accompany biofilm formation remains to be determined.

\section{ACKNOWLEDGEMENTS}

We thank Howard Jenkinson for providing strains, Sun Chung for technical assistance, and the NIDCR for support through DE12505, DE13061 and DE12750.

\section{REFERENCES}

Arai, M., Hamada, N. \& Umemoto, T. (2000). Purification and characterization of a novel secondary fimbrial protein from Porphyromonas gingivalis strain 381. FEMS Microbiol Lett 93, 75-81.

Belton, C. M., Izutsu, K. T., Goodwin, P. C., Park, Y. \& Lamont, 
R. J. (1999). Fluorescence image analysis of the association between Porphyromonas gingivalis and gingival epithelial cells. Cell Microbiol 1, 215-224.

Bradshaw, D. J., Marsh, P. D., Watson, G. K. \& Allison, C. (1998). Role of Fusobacterium nucleatum and coaggregation in anaerobe survival in planktonic and biofilm oral microbial communities during aeration. Infect Immun 66, 4729-4732.

Brooks, W., Demuth, D. R., Gil, S. \& Lamont, R. J. (1997). Identification of a Streptococcus gordonii SspB domain that mediates adhesion to Porphyromonas gingivalis. Infect Immun 65, 3753-3758.

Chung, W. O., Demuth, D. R. \& Lamont, R. J. (2000). Identification of a Porphyromonas gingivalis receptor for the Streptococcus gordonii SspB protein. Infect Immun 68, 6758-6762.

Chung, W. O., Park, Y., Lamont, R. J., McNab, R., Barbieri, B. \& Demuth, D. R. (2001). Signaling system in Porphyromonas gingivalis based on a LuxS protein. J Bacteriol 183, 3903-3909.

Cleary, P. \& Retnoningrum, D. (1994). Group A streptococcal immunoglobulin-binding proteins: adhesins, molecular mimicry or sensory proteins? Trends Microbiol 2, 131-136.

Cook, G. S., Costerton, J. W. \& Lamont, R. J. (1998). Biofilm formation by Porphyromonas gingivalis and Streptococcus gordonii. J Periodontol Res 33, 323-327.

Cornelis, G. \& Van Gijsegem, F. (2000). Assembly and function of type III secretory systems. Annu Rev Microbiol 54, 735-774.

Curtis, M. A., Kuramitsu, H. K., Lantz, M., Macrina, F. L., Nakayama, K., Potempa, J., Reynolds, E. C. \& Aduse-Opoku, J. (1999). Molecular genetics and nomenclature of proteases of Porphyromonas gingivalis. J Periodontol Res 34, 464-472.

Davies, D. G., Parsek, M. R., Pearson, J. P., Iglewski, B. H., Costerton, J. W. \& Greenberg, E. P. (1998). The involvement of cell-to-cell signals in the development of a bacterial biofilm. Science 280, 295-298.

Demuth, D. R., Berthold, P., Leboy, P. S., Golub, E. E., Davis, C. A. \& Malamud, D. (1989). Saliva-mediated aggregation of Enterococcus faecalis transformed with a Streptococcus sanguis gene encoding the SSP-5 surface antigen. Infect Immun 57, 1470-1475.

Demuth, D. R., Duan, Y., Brooks, W., Holmes, A. R., McNab, R. \& Jenkinson, H. F. (1996). Tandem genes encode cell-surface polypeptides SspA and SspB which mediate adhesion of the oral bacterium Streptococcus gordonii to human and bacterial receptors. Mol Microbiol 20, 403-413.

Demuth, D. R., Irvine, D. C., Costerton, J. W., Cook, G. S. \& Lamont, R. J. (2001). Discrete protein determinant directs the species-specific adherence of Porphyromonas gingivalis to oral streptococci. Infect Immun 69, 5736-5741.

Guggenheim, M., Shapiro, S., Gmur, R. \& Guggenheim, B. (2001). Spatial arrangements and associative behavior of species in an in vitro oral biofilm model. Appl Environ Microbiol 67, 1343-1350.

Hamada, N., Sojar, H. T., Cho, M.-I. \& Genco, R. J. (1996). Isolation and characterization of a minor fimbria from Porphyromonas gingivalis. Infect Immun 64, 4788-4794.

Hayashi, J., Nishikawa, K., Hirano, R., Noguchi, T. \& Yoshimura, F. (2000). Identification of a two-component signal transduction system involved in fimbriation of Porphyromonas gingivalis. Microbiol Immunol 44, 279-282.

Jenkinson, H. F. \& Demuth, D. R. (1997). Structure, function and immunogenicity of streptococcal antigen I/II polypeptides. Mol Microbiol 23, 183-190.

Jenkinson, H. F., Terry, S. D., McNab, R. \& Tannock, G. W. (1993). Inactivation of the gene encoding surface protein SspA in Streptococcus gordonii DL1 affects cell interactions with human salivary agglutinin and oral actinomyces. Infect Immun 61, 3199-3208.

Kinder, S. A. \& Holt, S. C. (1989). Characterization of coaggregation between Bacteroides gingivalis T22 and Fusobacterium nucleatum T18. Infect Immun 57, 3425-3433.

Kolenbrander, P. E. \& Andersen, R. N. (1989). Inhibition of coaggregation between Fusobacterium nucleatum and Porphyromonas (Bacteroides) gingivalis by lactose and related sugars. Infect Immun 57, 3204-3209.

Kolenbrander, P. E. \& London, J. (1993). Adhere today, here tomorrow: oral bacterial adherence. J Bacteriol 175, 3247-3252.

Lamont, R. J. \& Jenkinson, H. F. (1998). Life below the gum line: pathogenic mechanisms of Porphyromonas gingivalis. Microbiol Mol Biol Rev 62, 1244-1263.

Lamont, R. J. \& Jenkinson, H. F. (2000). Subgingival colonization by Porphyromonas gingivalis. Oral Microbiol Immunol 15, 341-349.

Lamont, R. J., Hersey, S. G. \& Rosan, B. (1992). Characterization of the adherence of Porphyromonas (Bacteroides) gingivalis to oral streptococci. Oral Microbiol Immunol 7, 193-197.

Lamont, R. J., Bevan, C. A., Gil, S., Persson, R. E. \& Rosan, B. (1993). Involvement of Porphyromonas gingivalis fimbriae in adherence to Streptococcus gordonii. Oral Microbiol Immunol 8, 272-276.

Lamont, R. J., Chan, A., Belton, C. M., Izutsu, K. T., Vasel, D. \& Weinberg, A. (1995). Porphyromonas gingivalis invasion of gingival epithelial cells. Infect Immun 63, 3878-3885.

Lee, S. W., Hillman, J. D. \& Progulske-Fox, A. (1996). The hemagglutinin genes hagG and hagC of Porphyromonas gingivalis are transcribed in vivo as shown by the use of a new expression vector. Infect Immun 64, 4802-4810.

Love, R. M., McMillan, M. D., Park, Y. \& Jenkinson, H. F. (2000). Coinvasion of dentinal tubules by Porphyromonas gingivalis and Streptococcus gordonii depends upon binding specificity of streptococcal antigen I/II adhesin. Infect Immun 68, 1359-1365.

Marsh, P. D. (1989). Host defenses and microbial homeostasis: role of microbial interactions. J Dent Res 68, 1567-1575.

Marsh, P. D. (1994). Microbial ecology of dental plaque and its significance in health and disease. Adv Dent Res 8, 263-271.

McNab, R. \& Jenkinson, H. F. (1998). Altered adherence properties of a Streptococcus gordonii hppA (oligopeptide permease) mutant result from transcriptional effects on $c s h A$ adhesin gene expression. Microbiology 144, 127-136.

Park, Y. \& Lamont, R. J. (1998). Contact-dependent protein secretion in Porphyromonas gingivalis. Infect Immun 66, 47774782.

Rosan, B. \& Lamont, R. J. (2000). Dental plaque formation. Microbes Infect 2, 1599-1607.

Scannapieco, F. A. (1994). Saliva-bacterium interactions in oral microbial ecology. Crit Rev Oral Biol Med 5, 203-248.

Schwarz, S., Ellen, R.P. \& Grove, D. A. (1987). Bacteroides gingivalis-Actinomyces viscosus cohesive interactions as measured by a quantitative binding assay. Infect Immun 55, 2391-2397.

Shaniztki, B., Hurwitz, D., Smorodinsky, N., Ganeshkumar, N. \& Weiss, E. I. (1997). Identification of a Fusobacterium nucleatum PK1594 galactose-binding adhesin which mediates coaggregation with periopathogenic bacteria and hemagglutination. Infect Immun 65, 5231-5237.

Socransky, S. S. \& Haffajee, A. D. (1992). The bacterial etiology of destructive periodontal disease: current concepts. J Periodontol 63, 322-331. 
Travis, J., Banbula, A. \& Potempa, J. (2000). The role of bacterial and host proteinases in periodontal disease. Adv Exp Med Biol 477, 455-465.

Wirth, R., An, F. Y. \& Clewell, D. B. (1986). Highly efficient protoplast transformation system for Streptococcus faecalis and a new Escherichia coli-S. faecalis shuttle vector. J Bacteriol 165, 831-836.

Xie, H., Chung, W. O., Park, Y. \& Lamont, R. J. (2000). Regulation of the Porphyromonas gingivalis fimA (fimbrillin) gene. Infect Immun 68, 6574-6579.
Yao, E. S., Lamont, R. J., Leu, S. P. \& Weinberg, A. (1996). Interbacterial binding among strains of pathogenic and commensal oral bacterial species. Oral Microbiol Immunol 11, 35-41.

Yoshimura, F., Takashi, K., Nodasaka, Y. \& Suzuki, T. (1984). Purification and characterization of a novel type of fimbriae from the oral anaerobe Bacteroides gingivalis. J Bacteriol 160, 949-957.

Received 23 January 2002; revised 12 February 2002; accepted 18 February 2002. 